\begin{tabular}{|c|c|}
\hline 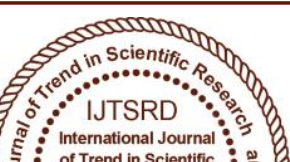 & $\begin{array}{l}\text { International Journal of Trend in Scientific } \\
\text { Research and Development (IJTSRD) }\end{array}$ \\
\hline 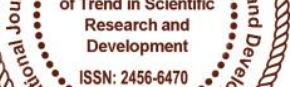 & International Open Access Journal \\
\hline intivis & ISSN No: 2456 - 6470 | www.ijtsrd.com | Volume - 2 | Issue -3 \\
\hline
\end{tabular}

\title{
An intelligent device control system using augmented reality and zigbee technology
}

\author{
Devi. $\mathbf{M}^{1}$, Renukasrinidhi. $\mathrm{N}^{2}$, Swarnamaalika. $\mathrm{N}^{3}$, Mrs. P. Rekha ${ }^{4}$ \\ ${ }^{1,2,3}$ B.E. Final Year, ${ }^{4}$ Assistant Professor, \\ Department of Computer Science Engineering, Sri Muthukumaran Institute of Technology, \\ Chikkarayapuram, Chennai, Tamil Nadu, India
}

\section{ABSTRACT}

Current technology and trend have made designing and implementation of embedded systems for home automation has increased the capabilities and features. There is a demand for smart home automation access via virtual reality. The proposed system allows the owner to access via virtual reality and controls the home appliances. This is very easy to access. In this paper, we present the design and implementation of a low cost but yet flexible, feasible and secure virtual reality based home automation system. In our proposed system we will try to control light, motor and 230v supply. This work also demonstrates the use of virtual reality in the context of the appliance control. In this project we have used image processing techniques for image acquisition, pre processing, image segmentation and feature extraction. The user commands are captured by the camera and transmitted through wireless communication to the receiver end. Thus our project has a huge scope for training and development business using virtual image and setting up a virtual environment.

Keywords: Virtual Reality Sensing Unit, Artificial Intelligence, Micro Controller AT89S52, Camera, Keil IDE, Embedded C

\section{INTRODUCTION}

The concepts behind virtual reality are based upon theories about a long held human desire to escape the boundaries of the 'real world' by embracing cyberspace. Once there we can interact with this virtual environment in a more naturalistic manner which will generate new forms of human-machine interaction (HMI).

The aim is to move beyond standard forms of interaction such as the keyboard and mouse which most people work with on a daily basis. This is seen as an unnatural way of working which forces people to adapt to the demands of the technology rather than the other way around. But a virtual environment does the opposite. It allows someone to fully immerse themselves in a highly visual world which they explore by means of their senses. This natural form of interaction within this world often results in new forms of communication and understanding.

Home automation is one of the major applications which is emerging. Home automation system is implemented using Bluetooth technology. Its frequency is of $2.4 \mathrm{GHz}$. It can link devices within $10 \mathrm{~m}$ to $100 \mathrm{~m}$ at a speed of $3 \mathrm{Mbps}$. With this capability of Bluetooth, we propose a home automation system based on Bluetooth technology.

There exist few concerns while designing a home automation system, Integrity and scalability plays a vital role. Also it should provide user-friendly interface. It includes few challenges like devices can be easily setup, monitored and controlled. This interface should also provide some diagnostic services so that if there is any problem with the system, it can be tracked down. Moreover the overall system should be fast enough to realize the true power of wireless technology. Finally the system should be cost effective in order to justify its application in home 
automation. Neng has presented and architecture for home automation where the system was based on a dedicated network. This system only shows how to solve home automation problems at software level and no hardware aspects were considered. Yavuz and Hasan presented a telephone and PIC based remote control system where pin- check algorithm has also been introduced. Also to remote control of home appliances such as oven, air conditioner and computer by telephones which offer easy usage has been investigated. Communication takes place via a dedicated telephone line not via a Bluetooth technology. Other studies such as one presented has examples of web based automation. However, they are not too feasible to be carried out as a low cost solution. Lately Al-Ali and AL-Rousan introduced a low cost Java- Based Home Automation System, without highlighting the low level details of the type of peripherals that can be attached.

\section{EXISTING SYSTEM}

The existing systems concerns about designing a home automation system, integrity and scalability plays a vital role. Also it should provide user-friendly interface. It includes few challenges like devices can be easily setup, monitored and controlled. This interface should also provide some diagnostic services so that if there is any problem with the system, it can be tracked down. It include internet based control systems which needs network connectivity and costlier. System based controlling is only done using Radio Frequency, Zigbee. In this huge data transmission is restricted. The issues with the existing system are external devices like personnel computers, smart phones are needed to access the devices.

\section{PROPOSED SYSTEM}

This proposed system allows the owner to access via virtual reality and controls the home appliances which are very easy to access. In this proposed system, we present the design and implementation of a low cost but yet flexible, feasible and secure virtual reality based home automation system. Here we will try to control light, motor and 230v supply. This work also demonstrates the use of virtual reality in the context of the appliance control. It uses image processing techniques for image acquisition, pre processing, image segmentation and feature extraction. The user commands are captured by the camera and transmitted through wireless communication to the receiver end. Thus the project has a huge scope for training and development business using virtual image and setting up a virtual environment.

\section{BLOCK DIAGRAM}

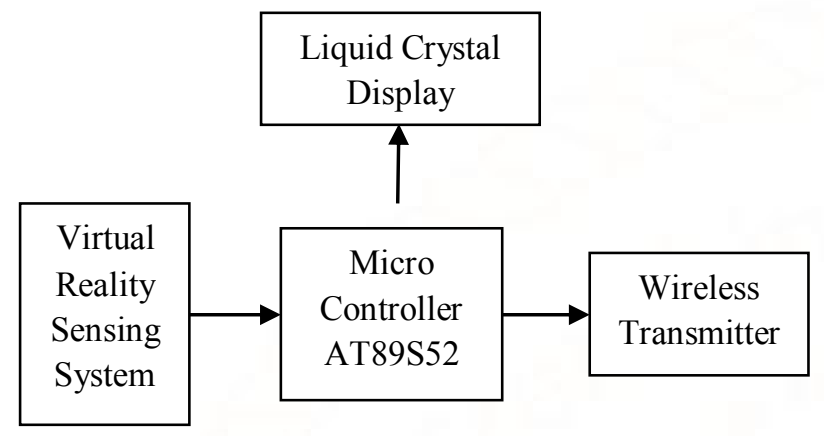

Fig. 1 Transmitter section

The Transmitter section consists of virtual reality sensing system, liquid crystal display and wireless transmitter interfaced with the micro controller AT89S52. Here the input is fetched from virtual reality sensing system and transmitter to the receiver section via wireless transmitter such as $\mathrm{ZigBee}$.

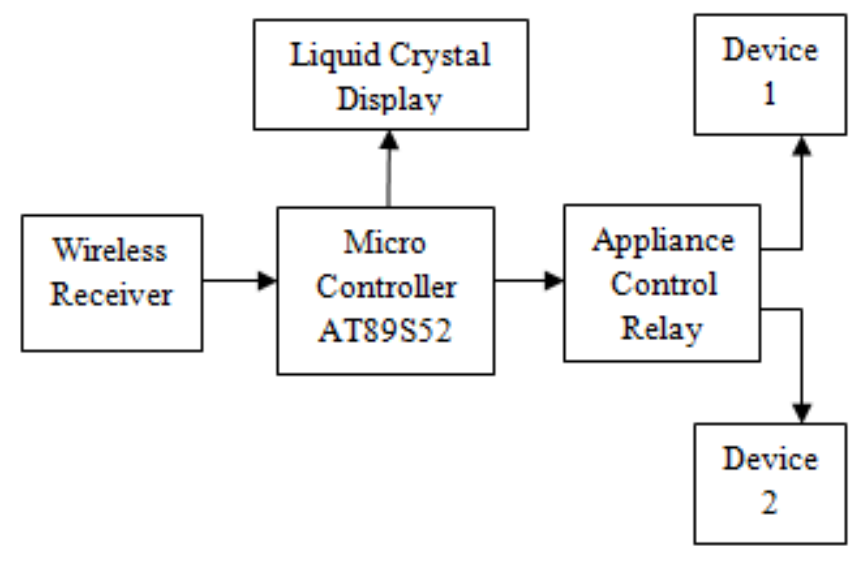

\section{Fig. 2 Receiver section}

The Receiver section consists of appliance control relay to control the connected devices, liquid crystal display and wireless receiver interfaced with the micro controller AT89S52. Here the fetched input is processed with the help of micro controller and the corresponding actions are taken via appliance control relay.

\section{WORKING}

Virtual Reality board designed to meet new forms of human-machine interaction (HMI). The advantage that enable the embedded system designer to easily, quickly and seamlessly add with the microcontroller by UART to their applications. Virtual Reality board can easily interface with PC or Laptop by the USB driver provided. By running the setup application, you 
can adjust the distance by viewing the camera image available in the PC or Laptop in which the optimize interaction can done.

\subsection{Virtual Reality Sensing System}

A hologram is a photographic recording of a light field, from an image formed by a lens. Every point in a hologram catches light waves that travel from every point in the object. That means wherever you look at a hologram you see exactly how light would have arrived at that point if you'd been looking at the real object. So a hologram is effectively a permanent record of what something looks like seen from any angle.

\subsection{Micro Controller AT89S52}

The AT89S52 is a low-power, high-performance CMOS 8-bit microcontroller with $8 \mathrm{~K}$ bytes of insystem programmable Flash memory. The device is manufactured using Atmel's high-density nonvolatile memory technology and is compatible with the Industry-standard 80C51 instruction set and pin out. The on-chip Flash allows the program memory to be reprogrammed in-system or by a conventional nonvolatile memory pro-grammer. By combining a versatile 8-bit CPU with in-system programmable Flash on a monolithic chip, the Atmel AT89S52 is a powerful microcontroller which provides a highlyflexible and cost-effective solution to many embedded control applications.

The AT89S52 provides the following standard features: $8 \mathrm{~K}$ bytes of Flash, 256 bytes of RAM, $32 \mathrm{I} / \mathrm{O}$ lines, Watchdog timer, two data pointers, three 16-bit timer/counters, a six-vector two-level interrupt architecture, a full duplex serial port, on-chip oscillator, and clock circuitry. In addition, the AT89S52 is designed with static logic for operation down to zero frequency and supports two software selectable power saving modes. The Idle Mode stops the CPU while allowing the RAM, timer/counters, serial port, and interrupt system to continue functioning. The Power-down mode saves the RAM con-tents but freezes the oscillator, disabling all other chip functions until the next interrupt or hardware reset. Finally devices are been controlled based on the user commands..

\subsection{Wireless Communication}

ZigBee is a specification for a suite of high level communication protocols using small, low-power digital radios based on the IEEE 802.15.4-2003 standard for wireless personal area networks (WPANs), such as wireless headphones connecting with cell phones via short-range radio. The technology defined by the ZigBee specification is intended to be simpler and less expensive than other WPANs, such as Bluetooth. ZigBee is targeted at radio-frequency (RF) applications that require a low data rate, long battery life, and secure networking.

\subsection{Appliance Control Relay}

A relay is an electrically operated switch. Many relays use an electromagnet to mechanically operate a switch, but other operating principles are also used, such as solid-state relays. Relays are used where it is necessary to control a circuit by a low-power signal or where several circuits must be controlled by one signal. In our project, we have used 4 SPDT relays in order to operate the two DC motors of the wheelchair..

\section{CONCLUSIONS}

A Device Control Based on Wireless Virtual Reality Technique using Artificial Intelligence has been \$yccessfully constructed to access via virtual reality and controls the home appliances which are very easy to access. This system is flexible, feasible and secure virtual reality based home automation system with cost efficient. Thus the proposed system has a huge scope for training and development business using virtual image and setting up a virtual environment.

\section{ACKNOWLEDGEMENT}

The authors give thanks to the project advisor, Mrs. P. Rekha, for her valuable input and guidance. Also we give thanks to our head of department, Dr. G. Rajinigirinath, for coordinating our project and giving valuable remarks. These people were essential to the success of this project.

\section{REFERENCES}

1. A.R.Al-Ali, M. AL-Rousan, "Java-Based Home Automation System". IEEE Transaction on ConsumerElectronics, Vol.50, No. 2, May 2004.

2. Neng-Shiang Liang, Li-Chen $\mathrm{Fu}, \mathrm{Chao-Lin} \mathrm{Wu}$, "An Integrated, Flexible, And Internet-Based Control Architecture For Home Automation System In The Internet Era", Proceedings ICRA'02. IEEE International Conference on Robotics and Automation, Vol. 2, pp.1101-1106, 2002. 
3. E. Yavuz, B. Hasan, I. Serkan, K. Duygu. "Safe and Secure PIC Based Remote Control Application for Intelligent Home", International Journal of Computer Science and Network Security, Vol. 7, No. 5, May 2007.

4. B. Koyuncu, "PC Remote Control Of Appliances By Using Telephone Lines", in IEEE Transaction on ConsumerElectronics, Vol. 41, Issue 1, pp.201209, 2005.

5. Kwang Yeol Lee, Jae Weon Choi, "RemoteControlled Home Automation System via Bluetooth Home Network", SICE Annual Conference in Fukui, August 4-6, 2003.

6. K.Tan, T.Lee, C.YeeSoh, "Internet-Based Monitoring of Distributed Control Systems - An Undergraduate Experiment". IEEE Transaction on Education, Vol. 45, No. 2, May 2002.

7. N. Swamy, O. Kuljaca, F. Lewis. "Internet-Based Educational Control Systems Lab Using Netmeeting", IEEE Transaction on Education, Vol. 45, No. 2, pp.145- 151, May 2002.

8. Ibari Benaoumeur, Ahmed-foitih Zoubir, Hanifi Elhachimi Amar Reda, "Remote Control of Mobile Robot using the Virtual Reality", in International Journal of Electrical and Computer Engineering (IJECE) Vol. 5, No. 5, October 2015, pp. 1062-1074.

9. Baki Koyuncu, "PC Remote Control Of Appliances By Using Telephone Lines", in IEEE Transactions on Consumer Electronics, Vol. 41, No. 1, FEBRUARY 2005, pp.201-209.

10. Selma Dilek, Hüseyin Çakır, Mustafa Aydın, "Applications Of Artificial Intelligence Techniques To Combating Cyber Crimes: A Review" in International Journal of Artificial Intelligence \& Applications (IJAIA), Vol. 6, No. 1, January 2015 